\title{
Author Correction: LRIG1 is a pleiotropic androgen receptor-regulated feedback tumor suppressor in prostate cancer
}

Qiuhui Li, Bigang Liu, Hsueh-Ping Chao, Yibing Ji, Yue Lu, Rashid Mehmood, Collene Jeter, Taiping Chen (D), John R. Moore, Wenqian Li, Can Liu, Kiera Rycaj, Amanda Tracz, Jason Kirk, Tammy Calhoun-Davis, Jie Xiong, Qu Deng, Jiaoti Huang, Barbara A. Foster, Abhiram Gokhale (1), Xin Chen \& Dean G. Tang (1)

Correction to: Nature Communications https://doi.org/10.1038/s41467-019-13532-4, published online 2 December 2019.

The original version of this Article contained an error in the third subheading of the "Results" section, which incorrectly read "Knocking down endogenous LRIG1 inhibits $\mathrm{AR}^{+} \mathrm{LRIG1}{ }^{+} \mathrm{PCa}$ ". The correct version should have been "promotes" in place of "inhibits".

This has been corrected in both the PDF and HTML versions of the Article.

Published online: 04 June 2020

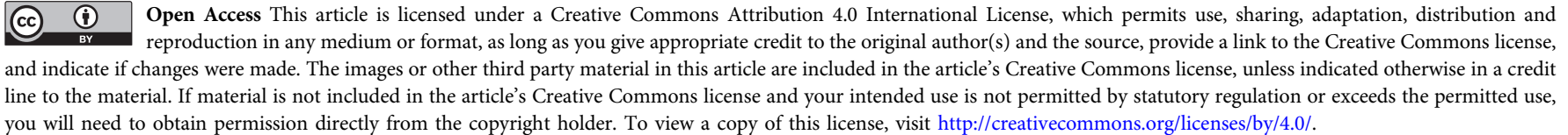
you will need to obtain permission directly from the copyright holder. To view a copy of this license, visit http://creativecommons.org/licenses/by/4.0/.

(C) The Author(s) 2020 